\title{
Self-assembled Lipid Structures as Model Systems for Studying Electrical and Mechanical Properties of Cell Membranes
}

\author{
Kaori Sugihara*
}

\begin{abstract}
This mini review summarizes our recent works in the development of electrical and mechanical characterization tools for cell membranes. These research topics require the application of physical and chemical characterization tools to biological systems, thus are very interdisciplinary.
\end{abstract}

Keywords: Electrochemistry · Lipid nanotube $\cdot$ Lipid self-assembly $\cdot$ Mechanochromic polymer · Pore-forming peptide

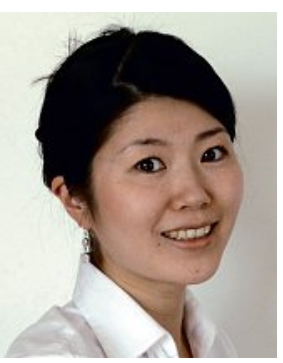

\section{Introduction}

Cell membranes separate cells from the outer world. All the information that comes from outside of our bodies (e.g. light, smell, sound) is converted into chemical or electrical signals at the cell membranes. They control many important physiological functions such as the five senses, ${ }^{[1]}$ muscle contractions, ${ }^{[2]}$ brain activities ${ }^{[3]}$ etc. Cell membranes are mainly composed of lipid bilayers and membrane proteins. Membrane proteins are notoriously difficult to study since they cannot be isolated easily. They retain their original functions only if they are kept incorporated inside the right lipid bilayer environment. That is why there are still many important membrane proteins that have not yet been well characterized.

My research focuses on understanding the role of lipids in cell membranes, the interaction between lipid bilayers and peptides, and the function of membrane proteins. Especially we are interested in their electrical and mechanical properties. Traditional biological approaches have limitations in addressing these aspects. Therefore, we have to adapt characterization tools in physics and chemistry (e.g. electrical measurements, atomic force microscopy, spectroscopy techniques) to biological samples for such investigations. Often the characterization technique does not even exist for what we want to study, thus we have to develop the setup ourselves. In this mini review, I summarize our recent works in the development of electrical and mechanical characterization tools for cell membranes and studies on peptide-membrane interactions using those methods.

\section{Development of High-throughput Electrophysiological Assays}

In my early career, we were developing a high-throughput platform for ion channel sensing. Ion channels are membrane proteins that regulate ion concentrations inside cells. Upon ligand binding or electrical, mechanical and optical stimuli, they transport certain types of ions from one side of cell membranes to the other. They have become prime drug targets in pharmaceutical industries as their key role in brain function is linked to many psychological diseases. ${ }^{[4]}$ The current golden standard technique to study these ion channels is the patch-clamp method. ${ }^{[5]}$ In this method, part of the cell membrane is 'patched' by a glass micropipette, and the ion current through the channels in the patch is monitored electrically. The technique has revolutionized the field and has answered many important biological questions in the last few decades as highlighted by the Nobel Prize awarded to Neher and Sakmann in 1991. However, the patch clamp technique is extremely labor intensive and the successful measurements often rely on experienced experimentalists' personal skills. Therefore, the adaptation of the technique to the high-throughput drug screening process is extremely challenging. The main difficulties come from the creation of a proper patch with a tight seal between the glass pipette and the cell membranes. Although it may sound trivial, this tight seal (called a 'giga-ohm seal') is the true novelty of the technique, which suppresses the background leakage current and boosts the sensitivity. There have been attempts to automate the patch clamp technique for high-throughput applications, ${ }^{[6]}$ however, such instruments also suffer from lack of reproducible patch formation, thus have a limited success. 
To overcome this problem, we developed a high-throughput ion channel sensing platform based on a synthetic bottomup approach (Fig. 1A). [7] The platform consists of a silicon nitride thin membrane with a polyelectrolyte-filled nanopore $(\phi=800 \mathrm{~nm}),{ }^{[8]}$ where a lipid bilayer is self-assembled over the pore by vesicle fusion. The approach enables the formation of tightly-sealed bilayers by self-assembly, eliminating the labor-intensive process in the traditional patch clamp method, manual patching. It makes the system highthroughput and reproducible. We incorporated a pore-forming peptide melittin, and monitored its single channel activities with inserted $\mathrm{Ag} / \mathrm{AgCl}$ electrodes over a few weeks (Fig. 1B). Such an extreme high stability (100 times longer bilayer lifetime compared to that of the traditional patch clamp method) is attributed to the underlying polymer that supports the bilayer mechanically. Another advantage is that the system contains only the target channel. The approach using native cell membranes has many other membrane proteins, which makes the interpretation of the data difficult. Such a synthetic system based on self-assembly can be used as an alternative electrophysiological assay for highthroughput drug screening.
The lipid bilayer-polymer composite used in the above-mentioned work is convenient, because it allows for the formation of bilayers on any substrate. Spontaneous planar lipid bilayer formation by vesicle fusion occurs only on limited types of substrates such as $\mathrm{SiO}_{2}$ and $\mathrm{TiO}_{2}$ but not on conductive surfaces such as gold. The mechanism of the vesicle fusion and the condition that allows for the bilayer formation are the important fundamental research topics that have attracted many researchers over the last decade. ${ }^{[9]}$ Nevertheless, one can coat such conductive substrates with cationic polyelectrolytes and enable bilayers to be formed by fusing anionic vesicles, using electrostatic interaction. ${ }^{[10]}$ Using this technique, we fabricated a bilayer over indium tin oxide (ITO)-coated waveguide of optical waveguide lightmode spectroscopy (OWLS, Fig. 2A). ${ }^{[11]}$ OWLS is a surface sensor that detects adsorbed mass by evanescent field. The top surface of its waveguide can be coated with ITO (conductive and optically transparent material), so that one can combine electrochemical characterization. We added melittin to the bilayer from the solution and studied the activity of melittin channels (Fig. 2B). The combined setup enables the monitoring of mass and the bilayer resistance simultaneously

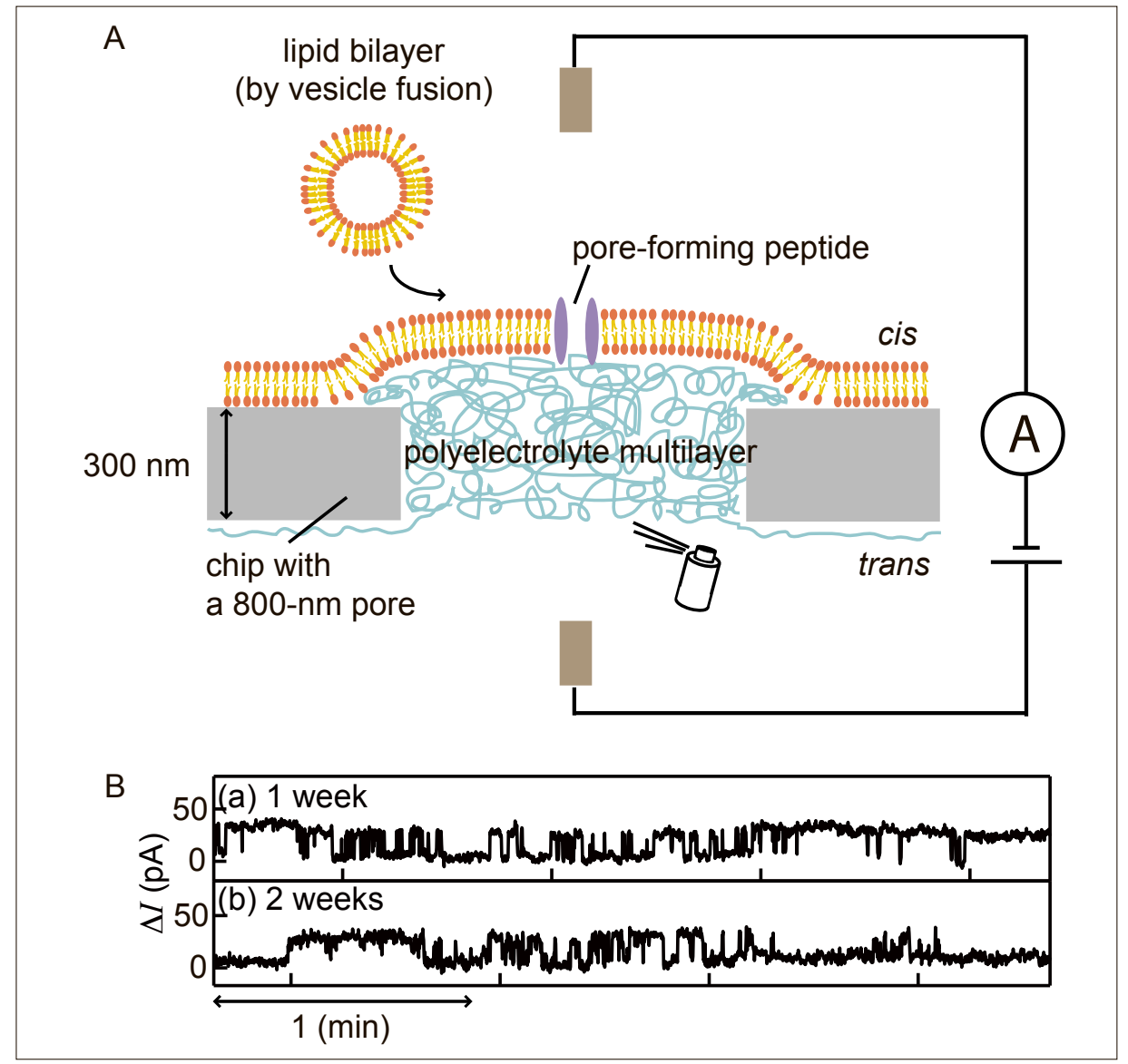

Fig. 1. A Gigaseal obtained with a self-assembled long-lifetime lipid bilayer on a single polyelectrolyte multilayer-filled nanopore. (A) A scheme presenting the design of the platform. (B) Single ion channel measurements from a melittin pore over a few weeks. Reprinted with permission from ref. [7]. Copyright 2010 ACS.

over time. It provides the correlation between the amount of melittin in the bilayer and their total channel size. It indicated that only a small fraction of melittin contributed to form pores, while most of them were simply adhered to the bilayer without forming channels. Such a combined setup is a powerful tool for the quantitative studies of the peptide-membrane interactions.

\section{Self-assembly of Lipid Nanotubes}

On the way to fabricate the bilayerpolyelectrolyte composite, I have accidentally discovered a new type of lipid nanotube self-assembly. I was trying to form defect-free bilayers over polyelectrolytes by varying the types of lipids and polyelectrolytes. Surprisingly, the combination of 1,2-dioleoyl-sn-glycero-3-phosphoethanolamine (DOPE, the main phospholipid found in bacteria) and polyethylenimine (PEI, a cationic polyelectrolyte) produced a network of lipid nanotubes as shown in the fluorescent image in Fig. 3A.[12] I still remember the shock I had during the experiment when I saw this unexpected fluorescence microscopy image. It was the beginning of my journey for the next several years to understand its self-assembling mechanism.

The assembly starts with formation of inverted hexagonal lipid blocks in physiological buffer solution. DOPE is a conical lipid that prefers the inverted hexagonal phase, where the inverted micelle tubes are bundled into a hexagonal pattern, constructing a densely-packed three-dimensional architecture (Fig. 3B). ${ }^{[13]}$ These inverted hexagonal lipid blocks were attached to PEI-coated glass slides. Later on, we found out that any other surfaces that permit the 'weak' adsorption of the lipid blocks enables the lipid nanotube self-assembly in a similar way. When the interaction between the substrate and the lipid blocks is too strong, lipids typically spread all over the surfaces and hinder the next step for the assembly. When there is a fluctuation in the solution such as turbulence flow created by pipettes or lamellar flow induced by microfluidic systems, a part of the lipid block moves while the other part is kept attached to the substrates, pulling out a tube as shown in the cryo-transmission electron microscopy (cryoTEM) image in Fig. 3C. In the field of membrane physics, there is the standard lipid nanotube system created from a giant unilamellar vesicle (a singlebilayer vesicle with a diameter of 1-10 $\mu \mathrm{m})$ by applying a point load with a glass micropipette connected to a micromanipulator. ${ }^{[14]}$ After all, the fundamental mechanism how the lipid nanotubes form in our system is somewhat similar to the one with 


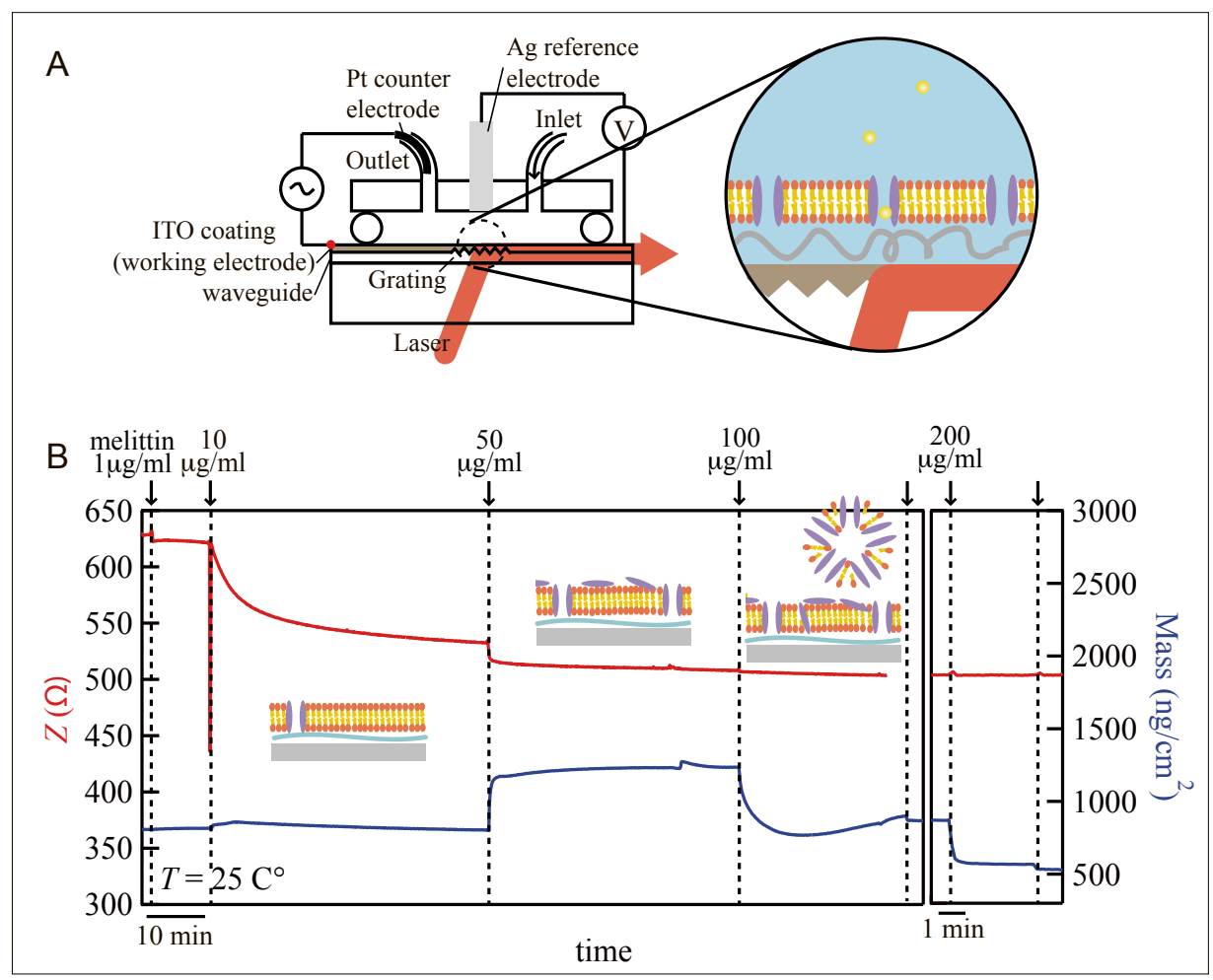

Fig. 2. Simultaneous OWLS (optical waveguide lightmode spectroscopy) and EIS (electrochemical impedance spectroscopy) monitoring of supported lipid bilayers with the pore forming peptide melittin. (A) A scheme showing the setup. (B) Impedance and adsorbed mass monitored simultaneously while adding melittin to bilayers. Reprinted with permission from ref. [9]. Copyright 2012 Elsevier.
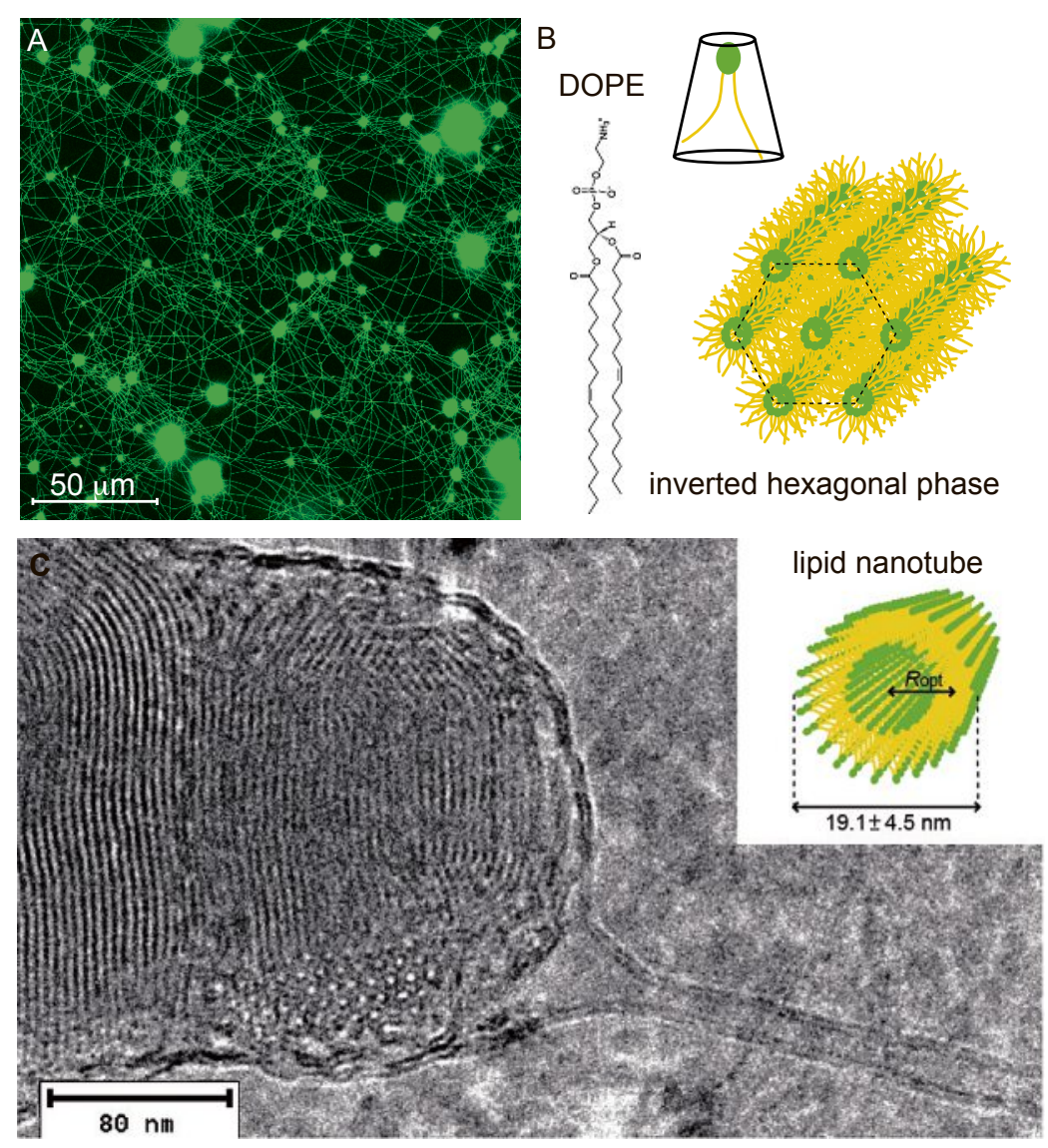

Fig. 3. Directed self-assembly of lipid nanotubes from inverted hexagonal structures. (A) A fluorescent image of the surface-assembled lipid nanotubes. (B) The chemical structure of DOPE lipid and a scheme of the inverted hexagonal phase. (C) A cryoTEM image of the lipid nanotube protruding from the inverted hexagonal lipid block. Reprinted with permission from ref. [12]. Copyright 2012 ACS. giant unilamellar vesicles; the application of the point load to the membrane structure. However, in our system, the point load is not explicitly applied by a pipette. The shear force induced by solution flow is enough to protrude tubes, resulting in their self-assembly all over the surfaces without manual manipulation.

I found this phenomenon extremely interesting, because at the molecular level the difference between our DOPE lipids that create self-assembled lipid nanotubes and the other lipids that do not could be as small as the presence of one double bond. For example, POPE is a phospholipid that has an identical head group as DOPE with one double bond in its tail. It does NOT selfassemble into lipid nanotubes. On the other hand, the same PE lipids with an additional one double bond (DOPE) magically present self-assembly of lipid nanotubes. Why? It is because the presence of one double bond changes the phase of lipids at room temperature. POPE is a cylindrical lipid that takes a lamellar phase, thus it forms vesicles under aqueous solution. DOPE is much more conical due to the additional double bond that creates a kink in the tail, therefore prefers the inverted hexagonal phase. The lipid nanotube assembly starts with the inverted hexagonal lipid blocks, thus all the other lipids that take lamellar phases do not selfassemble tubes in the same way.

Next, why is the inverted hexagonal structure the precondition for the selfassembly of tubes? Inverted hexagonal structure is a unique phase where many lipids are packed in inverted micelle bundles within a bilayer envelope as shown in the cryoTEM image in Fig. 3C. We think that the key is these densely-packed lipids, which act as a reservoir for providing materials to form tubes. In such a lipid object that has a 'lipid reservoir', the increase in the surface area does not require stretching of the bilayers but can be performed simply by moving the lipids from the internal reservoir to the surface. In other words, in this system the origin of the surface tension is the lipid phase transformation from the inverted hexagonal to the lamellar structure, which is kept constant at relatively low value throughout the large deformation regime. The protrusion of lipid tubes increases the total surface area. Therefore, such an object with a relatively low surface tension has an advantage for forming tubes, because the associated energy penalty is small.

The low surface tension of inverted hexagonal lipid blocks can be also seen when we try to manipulate the lipid nanotubes by micromanipulator (Fig. 4). When a micropipette touches the pre-assembled lipid nanotubes (Fig. 4A,B) or surfaceattached lipid blocks (Fig. 4C,D), part of the lipid adheres to the glass micropipette. 

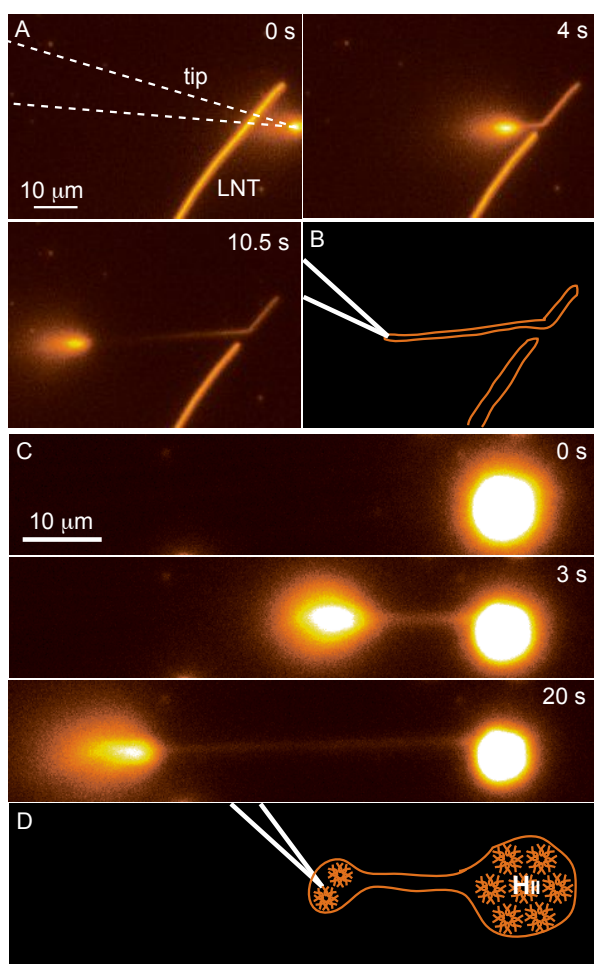

Fig. 4. Freely drawn single lipid nanotube patterns. A lipid nanotube formation from $(A, B)$ preassembled lipid nanotubes and $(C, D)$ from an inverted hexagonal lipid block. Reprinted with permission from ref. [15]. Copyright 2015 RSC.

If we keep pulling the pipette, we can simply form a new lipid nanotube from the existing one. ${ }^{[15]}$ If one attempts to create a tube from a giant unilamellar vesicle in a similar way, additional tricks such as voltage pulse application ${ }^{[16]}$ or incorporation of biotin in the vesicles and coating of the pipette with streptavidin ${ }^{[17]}$ are required to assure the reproducible connection between the vesicles and the pipette. This is because giant unilamellar vesicles do not adhere to the pipette otherwise, but rather try to dodge when one attempts to touch them due to their high surface tension.

These surface-assembled lipid nanotubes can be used to study cell contractility. A fibroblast was seeded on top of rhodamine-stained lipid nanotubes, and its initial spreading was monitored by fluorescence microscopy. ${ }^{[18]}$ Fig. 5B,C are the snapshots from the bright field and the rhodamine channel respectively. As the cell spreads, new lipid nanotubes came out from the edge of the cell. It looks as if the cell grabbed the existing lipid nanotubes and is pulling them towards the cell center. We found that these new lipid nanotubes are nucleated between focal adhesions (anchors that cells make to firmly attach substrates). Inhibition of the actin-myosin affinity by blebbistatin suppressed it. All the evidences suggest that this phenomenon is linked to the cell contractility. When initial small focal contacts try to make an anchor to the substrate, sometimes they at- tach to nanotubes instead of the solid substrate. Upon application of forces, these focal contacts slip because lipids are very soft, resulting in the nucleation of new lipid nanotube branches (Fig. 5A). In other words, cells are applying a point load to the lipids via focal contacts, somewhat similarly to the way we do it by micropipettes. From the fundamental studies, we know that the minimum force required to start pulling lipid nanotubes in our system is around $90 \mathrm{pN},{ }^{[12]}$ which corresponds to 20-30 myosin motors (a single myosin exceeds 3-4 pN). ${ }^{[19]}$ Using this approach, we studied the cell force activity of different types and conditions of cells in a labelfree manner. For example, malignant cells typically have low contractility, which was visualized by absence of the nucleation of new lipid nanotubes, while endothelial cells pulled the lipid nanotubes intensively, presenting a high level of contractility.

Another use of the lipid nanotubes is as a template for fabricating solid structures. As a proof of concept, we fabricated gold nanowires with the lipid nanotube templates by attaching gold nanoparticles to the lipid nanotubes, fixing them chemically, removing the template through oxygen plasma cleaning, and annealing. [20] Gold nanoparticles were attached to the lipid nanotubes, using streptavidin-biotin inter- action (Fig. 6A). Both cryoTEM (Fig. 6B) and atomic force microscopy (Fig. 6C) showed successful adhesion of gold nanoparticles to the template. Electrical conductivity was observed only after the oxygen plasma treatment for removing the organic template (Fig. 6D). The approach exploits the self-assembled organic template for the high-throughput nanostructure fabrication. The same strategy can be used to align other particles and biomolecules.

\section{Mechanochromic Crosslinked Lipids; Polydiacetylene (PDA)}

Since I started my independent group in Geneva in 2014, we started working on diacetylene. Diacetylene is a polymerizable lipid that one can self-assemble into different structures and can be subsequently crosslinked into a polymer (polydiacetylene, PDA) by UV. PDA has garnered attention due to its chromism reported in the late $70 s^{[21]}$ When the polymer is exposed to mechanical stress, change in temperature, $\mathrm{pH}$ etc., the structural perturbation can be seen by the change in its color ${ }^{[21]}$ or in its fluorescence intensity. ${ }^{[22]}$ On the other hand, PDA is electrically conductive, thus is once considered as a material for electronics applications. ${ }^{[23]}$ Although the material has been studied for the last

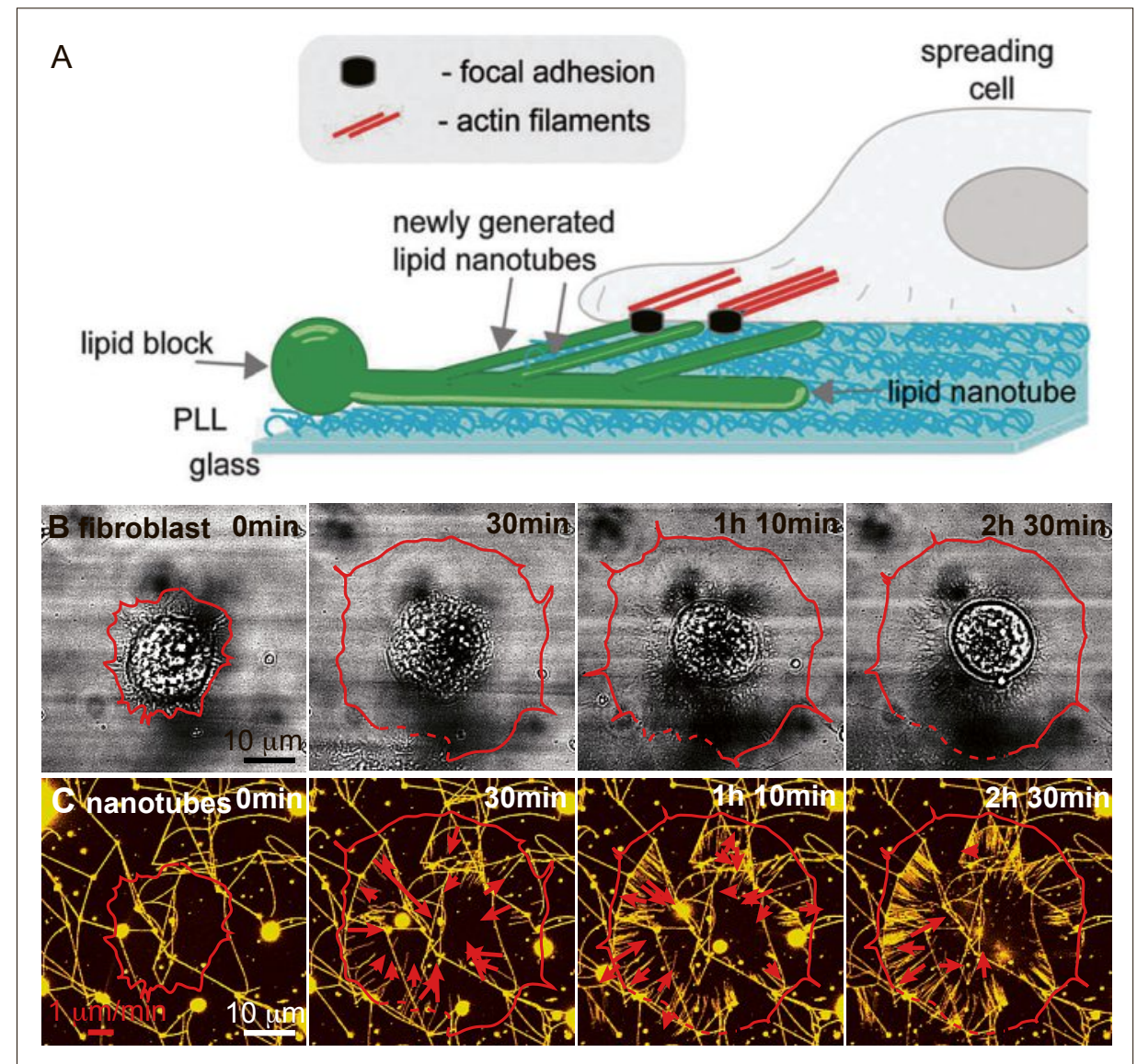

Fig. 5. Label-free detection of cell-contractile activity with lipid nanotubes. (A) A scheme showing the new lipid nanotube formation directed by cell forces. (B) Bright field and (C) fluorescent images from a movie where the interaction between a fibroblast and lipid nanotubes was monitored. Reprinted with permission from ref. [18]. Copyright 2013 RSC. 


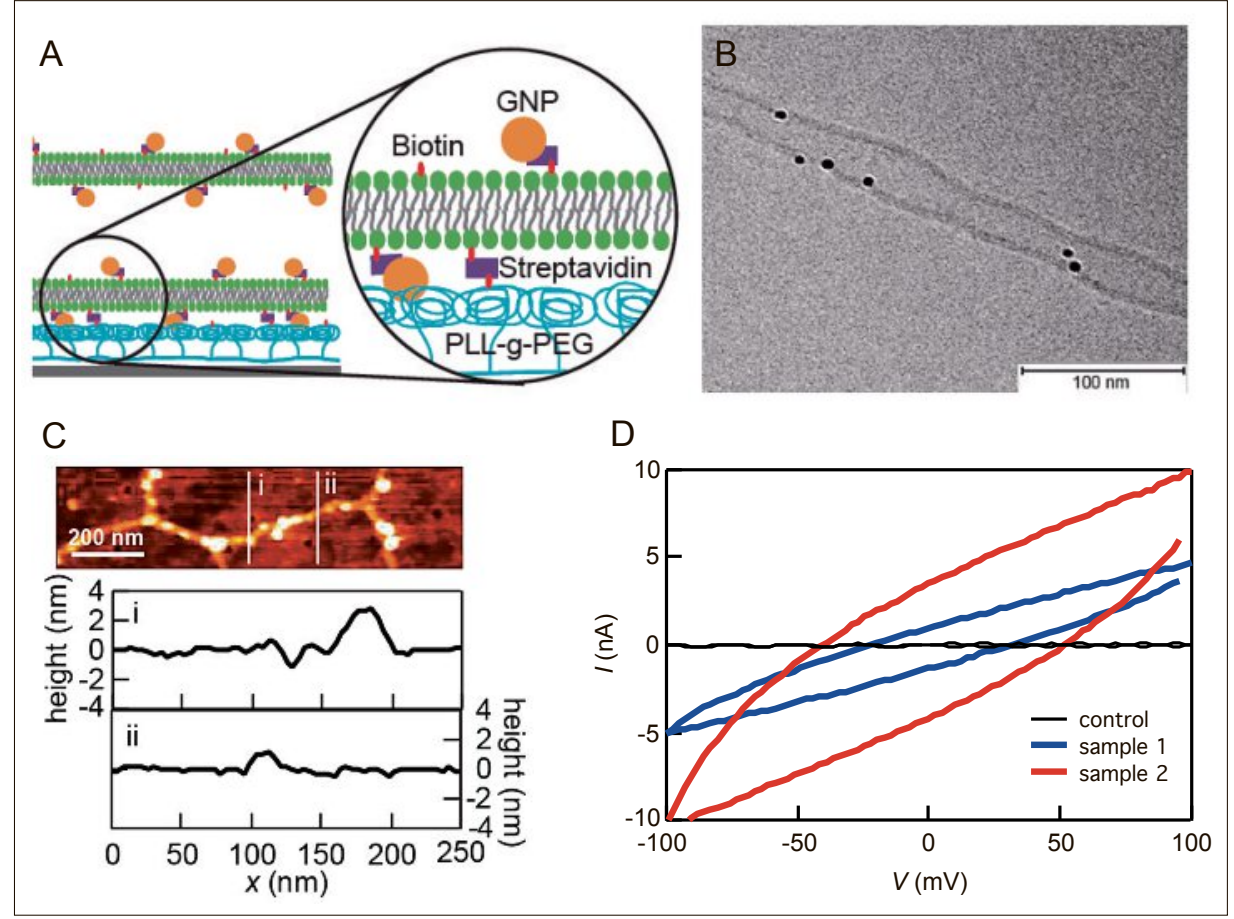

Fig. 6. Gold nanowire fabrication with surface-attached lipid nanotube templates. (A) A scheme describing the strategy of gold nanoparticle attachment to the lipid nanotubes. (B) A cryoTEM image of gold nanoparticle attached lipid nanotubes. (C) An AFM image of gold nanoparticle adhered lipid nanotubes after chemical fixing. The cross sections correspond to the white lines in the image. (D) $I-V$ curve from the fabricated gold nanowires. Reprinted with permission from ref. [20]. Copyright 2016 Wiley.

decades, its optical characterization in the field of chemistry and the electrical investigation in physics are not well correlated. We studied the properties of PDA made of 10,12-tricosadiynoic acid both by optical and electrical methods. ${ }^{[24]}$ We found a systematic decrease of PDA electrical conductance that coincides with the blue shift of the PDA absorption spectra upon UV irradiation (Fig. 7). The results can be comprehensively explained by the change in the electrical band gap. We have also shown that the PDA with a phospholipid headgroup (Diyne PC) changes its color when melittin is added in a concentration- dependent manner. It opens a possibility to use those PDAs as model cell membranes to detect peptides.

\section{Conclusion}

Lipid self-assembly attracts researchers due to its non-trivial fundamental mechanism. Understanding its detailed properties is also extremely useful for developing synthetic model cell membranes for investigating membrane active peptides and membrane proteins. As a future direction, we want to use these developed electrical and mechanical characterization

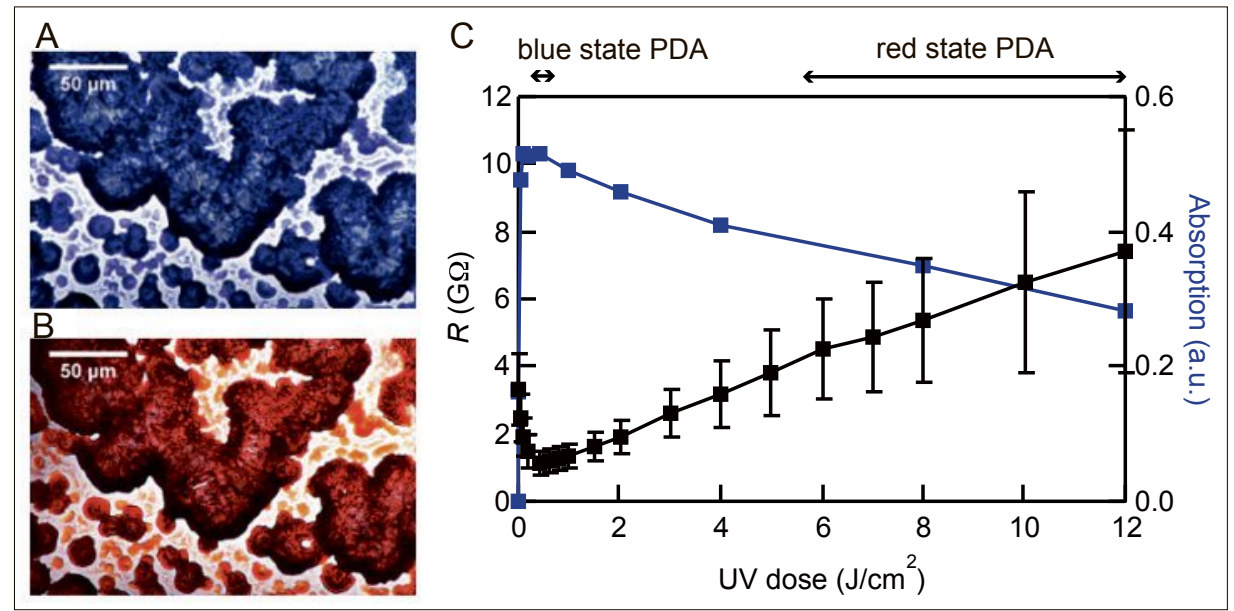

Fig. 7. Combined electrical and optical characterization of polydiacetylene. Bright field images of (A) blue and (B) red polydiacetylene. (C) The change in the electrical conductance of polydiacetylene coincides with the blue shift of the absorption spectra upon UV irradiation. Reprinted with permission from ref. [21]. Copyright 2016 ACS. tools to address biological questions such as the mechanism of the cooperativity of antimicrobial peptides and the function of ion channels.

\section{Acknowledgements}

I would like to thank all my past and current students for their tremendous work. I would like also to take this opportunity to thank my previous supervisors, Prof. Janos Vörös and Prof. Joachim P. Spatz. I thank the University of Geneva for giving me an opportunity to start my own group. The presented works were partially funded by Swiss National Science Foundation, Alexander von Humboldt Foundation, NCCR Chemical Biology, Novartis Foundation for Medical-Biological Research.

Received: August 10, 2016

[1] R. Syeda, J. Xu, A. E. Dubin, B. Coste, J. Mathur, T. Huynh, J. Matzen, J. Lao, D. C. Tully, I. H. Engels, H. M. Petrassi, A. M. Schumacher, M. Montal, M. Bandell, A. Patapoutian, Elife 2015, 4, DOI: 10.7554/eLife.07369.

[2] A. Noma, Nature 1983, 305, 147.

[3] R. Dingledine, K. Borges, D. Bowie, S. F. Traynelis, Pharmacol. Rev. 1999, 51, 7 .

[4] H. Mohler, Neuropharmacology 2012, 62, 42.

[5] O. P. Hamill, A. Marty, E. Neher, B. Sakmann, F. J. Sigworth, Pflügers Archiv Eur. J. Physiol. 1981, 391,85 .

[6] J. Dunlop, M. Bowlby, R. Peri, D. Vasilyev, R. Arias, Nat. Rev. Drug Discov. 2008, 7, 358.

[7] K. Sugihara, J. n. Vörös, T. Zambelli, ACS nano 2010, $4,5047$.

[8] K. Sugihara, J. n. Vörös, T. Zambelli, J. Phys. Chem. B 2010, 114, 13982

[9] a) K. Sugihara, B. Jang, M. Schneider, J. Voros, T. Zambelli, Soft Matter 2012, 8, 5525; b) E. Reimhult, M. Zach, F. Hook, B. Kasemo, Langmuir 2006, 22, 3313; c) R. Richter, A Brisson, Eur. Biophys. J. 2005, 34, 706.

[10] S. E. Moya, J. L. Toca-Herrera, J. Nanosci. Nanotechnol. 2006, 6, 2329.

[11] K. Sugihara, M. Delai, I. Szendro, O. GuillaumeGentil, J. Vörös, T. Zambelli, Sensors and Actuators B: Chem. 2012, 161, 600.

[12] K. Sugihara, M. Chami, I. Derenyi, J. Voros, T. Zambelli, ACS nano 2012, 6, 6626.

[13] K. Sugihara, J. Stucki, L. Isa, J. Vörös, T. Zambelli, J. Colloid Interface Sci. 2012, 386, 421.

[14] D. Cuvelier, N. Chiaruttini, P. Bassereau, P. Nassoy, Europhys. Lett. 2005, 71, 1015

[15] K. Sugihara, A. Rustom, J. P. Spatz, Soft Matter 2015, 11, 2029.

[16] A. Karlsson, R. Karlsson, M. Karlsson, A.S. Cans, A. Stromberg, F. Ryttsen, O. Orwar, Nature 2001, 409, 150

[17] G. Koster, A. Cacciuto, I. Derenyi, D. Frenkel, M. Dogterom, Phys. Rev. Lett. 2005, 94 , 068101.

[18] K. Sugihara, M. Delai, R. Mahnna, J. Kusch, D. Poulikakos, J. Voros, T. Zambelli, A. Ferrari, Integrative Biology: Quantitative Biosciences from Nano to Macro 2013, 5, 423.

[19] J. T. Finer, R. M. Simmons, J. A. Spudich, Nature 1994, 368, 113

[20] K. Jajcevic, M. Chami, K. Sugihara, Small 2016, DOI: $10.1002 / \mathrm{smll} .201600431$

[21] R. R. Chance, Macromolecules 1980, 13, 396.

[22] R. W. Carpick, D. Y. Sasaki, A. R. Burns, Langmuir 2000, 16, 1270.

[23] H. Nakanishi, F. Mizutani, M. Kato, K. Hasumi, J. Polym. Sci. Pol. Lett. 1983, 21, 983.

[24] C. Girard-Reydet, R. D. Ortuso, M Tsemperouli, K. Sugihara, J. Phys. Chem. B 2016, 120,3511 